\section{Basal Implantology}

Editor: Gérard M. Scortecci

Publisher: Springer Nature, Switzerland

Language: English

ISBN: 978-3-319-44873-2

Edition: 1/e

Publish Year: 2019

Pages: 398, illustrated

Price: $€ 117,69$

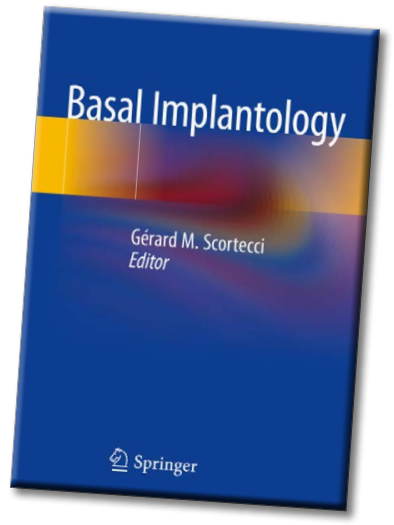

Marian-Vladimir

Constantinescu

$\mathrm{DDS}, \mathrm{MSc}, \mathrm{PhD}$

Holistic Dental \& Medical Institute

of Bucharest - ROPOSTURO

Bucharest, Romania

e-mail:

dr.vladimir.constantinescu@gmail.com

Dr. Scortecci, inventor of Diskimplant and holder of several associated patents, together with his 16 collaborators, drafted a book entitled Basal Implantology which explains the principles that underlie the use of basal implants. Diskimplants have been used in oral implantology for over 30 years as an alternative to more invasive procedures in desperate clinical situations and may represent a last chance for an oral invalid to have fixed teeth once again. This book is a guide to the practical application of biological, mechanical, and prosthetic principles of basal implantology and osseointegration (BIO concept), from simple solutions to complex clinical situations without the need for prior bone grafting.

The book is divided into three parts with fifteen chapters.

Part I, Fundamental Basis, has five chapters and explains the basic principles, biological aspects, biomechanics, evidence-based basal implantology and initial bone bed activation.

Part II, Step-by-Step Basal Implantology, has a more practical approach and in four chapters explains the indications and contraindications, how to establish treatment planning, surgery procedures and prosthetic stages. Finally, Clinical Applications and Complications, in six chapters exemplifies single-tooth replacement in the esthetic zone and posterior sectors, how to approach partial edentulism, completely edentulous, atrophic jaws and extreme clinical situations, presents multicenter clinical applications, complications and postimplantation neuropathies.

The book is a practical guide for implant surgeons and helps them find simple solutions to complex and highly demanding clinical situations. To facilitate understanding it is abundantly illustrated with pictures from clinical cases, CT's and radiographs.

Basal Implantology was written to support implantologists to successfully perform oral rehabilitation without more invasive procedures.

d.) http://www.stomaeduj.com 10.25241/stomaeduj.2020.7(2).bookreview.1

The Books Review is drafted in the reviewer's sole wording and illustrates his opinions. 